\title{
Long-term importance of right ventricular outflow tract patch function in patients with pulmonary regurgitation
}

\author{
Rajesh Puranik, MD, ${ }^{\text {a,b }}$ Victor Tsang, MD, ${ }^{\text {bc }}$ Philip Lurz, MD, ${ }^{\text {a,b }}$ Vivek Muthurangu, MD, ${ }^{\text {a,b }}$ \\ Sophie Offen, MBBS, ${ }^{\mathrm{a}}$ Alessandra Frigiola, MD, ${ }^{\mathrm{a}, \mathrm{b}, \mathrm{c}}$ Wendy Norman, DCR, ${ }^{\mathrm{a}}$ Fiona Walker, MD, \\ Philip Bonhoeffer, MD, ${ }^{\mathrm{a}}$ and Andrew M. Taylor, $\mathrm{MD}^{\mathrm{a}, \mathrm{b}}$
}

Objective: Chronic pulmonary regurgitation (PR) has deleterious effects on right ventricular (RV) function in repaired tetralogy of Fallot (ToF). However, there are little data regarding right ventricular outflow tract (RVOT) contractile dysfunction in response to chronic PR and on both RV and LV volumes and function.

Methods: We retrospectively identified consecutive patients with PR who were referred for magnetic resonance imaging quantification of "free PR" detected on echocardiography between 2003 and 2008. Patients had ToF and a transannular patch procedure $(\mathrm{n}=30,25.1 \pm 1.2$ years $)$ or PR resulting from valvar pulmonary stenosis treated with surgical or percutaneous valvotomy $(n=30,26.6 \pm 1.8$ years $)$.

\begin{abstract}
Results: The ToF and the PS groups were well matched for age at scan, age at repair surgery in ToF or initial valvotomy in PS, duration of exposure to PR, body surface area, heart rate, PR fraction, net forward pulmonary artery flow, and main and branch pulmonary artery dimensions. Severe PR fractions were identified in both groups (ToF: $40 \% \pm 1 \%$ vs PS: $37 \% \pm 2 \%, P=.2$ ). Indexed RV and LV end-diastolic volumes were similar for both ToF and PS groups (RV end-diastolic volume index: $137 \pm 6 \mathrm{~mL} / \mathrm{m}^{2}$ vs $128 \pm 5 \mathrm{~mL} / \mathrm{m}^{2}, P=.2$, and LV end-diastolic volume index: $72 \pm 2 \mathrm{~mL} / \mathrm{m}^{2}$ vs $67 \pm 2 \mathrm{~mL} / \mathrm{m}^{2}, P=.1$, respectively). RV mass was also similar between groups ( $95 \pm 5 \mathrm{~g}$ vs $81 \pm 6 \mathrm{~g}$, respectively, $P=.08$ ). However, indexed RV and LV end-systolic volumes were consistently higher in ToF when compared with PS (RV end-systolic volume index: $70 \pm 5 \mathrm{~mL} / \mathrm{m}^{2}$ vs $54 \pm$ $3 \mathrm{~mL} / \mathrm{m}^{2}, P<.01$, and LV end-systolic volume index: $29 \pm 1 \mathrm{~mL} / \mathrm{m}^{2}$ vs $22 \pm 1 \mathrm{~mL} / \mathrm{m}^{2}, P<.01$, respectively). These changes were reflected in lower biventricular systolic function in patients with ToF when compared with PS (RV ejection fraction: $52 \% \pm 1.5 \%$ vs $59 \% \pm 1 \%, P<.001$, and LV ejection fraction: $61 \% \pm 1 \%$ vs $67 \pm$ $1 \%, P<.001$, respectively). Although RV transannular plane systolic excursion was not significantly different between the groups $(P=.86)$, the RV outflow tract was considered contractile in only $50 \%$ of patients with ToF compared with $93 \%$ of patients with PS $(P=.0004)$. RV volumes and function were similar when only patients with contractile RV outflow tracts were compared.
\end{abstract}

Conclusions: RV outflow tract patch dysfunction in repaired ToF is responsible for higher end-systolic volumes and thus lower global measures of ventricular systolic function. These findings were not evident in cases of PS treated with valvotomy with comparable amount of PR. These observations highlight the importance of the initial repair surgery in ToF for late outcomes. (J Thorac Cardiovasc Surg 2012;143:1103-7)

Chronic pulmonary regurgitation (PR) has deleterious effects on right ventricular (RV) function. This is particularly true in repaired tetralogy of Fallot $(\mathrm{ToF}),{ }^{1-3}$ with both the

\footnotetext{
From the Centre for Cardiovascular Imaging, ${ }^{\text {a }}$ UCL Institute of Cardiovascular Science, and Cardiorespiratory Unit, ${ }^{\mathrm{b}}$ Great Ormond Street Hospital for Children, London, United Kingdom; and GURCH Unit, ${ }^{\mathrm{c}}$ The Heart Hospital, London, United Kingdom.

R. Puranik is the Neil Hamilton Fairley National Health Medical Research Council of Australia, Neil Hamilton Fairley Fellowship of Australia Clinical Overseas Postdoctoral fellow.

Disclosures: Authors have nothing to disclose with regard to commercial support.

Received for publication March 28, 2011; revisions received July 10, 2011; accepted for publication Sept 26, 2011; available ahead of print Nov 7, 2011.

Address for reprints: Andrew M. Taylor, MD, Director, Centre for Cardiovascular Imaging, UCL Institute Cardiovascular Science, Great Ormond Street Hospital for Children, Great Ormond St, London WC1N 3JH, United Kingdom (E-mail: a.taylor76@ucl.ac.uk).

$0022-5223 / \$ 36.00$

Copyright (c) 2012 by The American Association for Thoracic Surgery

doi:10.1016/j.jtcvs.2011.09.039
}

severity and ventricular response to PR determining longterm outcomes. ${ }^{4-7}$ However, patients with PR secondary to pulmonary stenosis (PS) treated with valvotomy have excellent long-term survival. Survival is similar to that of age-matched normal controls, ${ }^{8,9}$ markedly contrasting with repaired ToF. Thus, the presence of PR alone cannot explain the excess of morbidity and mortality in the population with ToF. There are 3 possible explanations for the differences between these patients with repaired ToF and PS. First, the 2 groups may have different amounts of PR. Second, operative remodeling of the RV outflow tract (RVOT) in ToF may have deleterious effects on later RV function. Last, there may be differences in the inherent RV contractility in these 2 groups.

The aim of this study was to investigate the factors that influence global ventricular function in patients with repaired ToF and PS. To control for the effect of different amounts of PR, age-matched patients were selected with 


\section{Abbreviations and Acronyms \\ $\mathrm{LV} \quad=$ left ventricular \\ PA $=$ pulmonary artery \\ PS = pulmonary stenosis \\ $\mathrm{PR} \quad=$ pulmonary regurgitation \\ $\mathrm{RV} \quad=$ right ventricular \\ RVOT $=$ right ventricular outflow tract \\ TAPSE $=$ transannular plane systolic excursion \\ TOF $=$ tetralogy of Fallot}

similar regurgitation fractions. Thus, the specific factors that were assessed were RVOT patch function and myocardial contractility. Investigating these factors may assist in our understanding of the impact of initial repair strategies and key determinants of long-term outcomes in these conditions.

\section{MATERIALS AND METHODS \\ Patient Population}

Between March 2003 and April 2008, 30 consecutive patients (aged $26.6 \pm 1.8$ years, 14 male/16 female) with free PR as a result of PS treated with surgical or percutaneous balloon valvotomy were identified.

In this PS group, 21 patients underwent percutaneous balloon valvotomy, with 5 patients requiring a second balloon valvotomy procedure. Nine patients underwent open surgical pulmonary valvotomy, which was combined with limited RVOT autologous pericardial patch surgery in 4 patients. In all patch procedures, the pulmonary valve/annulus was spared. In 6 of the 9 patients undergoing surgical valvotomy, concomitant repair of secundum atrial septal defects was performed. In follow-up, 4 patients in the PS group were identified to have PR in combination with mild $(\mathrm{n}=3)$ or moderate $(\mathrm{n}=1)$ RVOT obstruction.

The patients with PS were age and PR fraction matched, with 30 patients followed in the Adult Congenital Heart Disease clinical service with repaired ToF (aged $26.6 \pm 1.8$ years, 18 male/12 female) and in whom "free PR" had also been identified. All patients with ToF had undergone a transannular autologous pericardial patch procedure in childhood. In addition, 9 of these patients had undergone a palliative shunt procedure in infancy ( 8 with modified Blalock-Taussig shunt procedures and 1 with a Waterston's shunt). Two patients in the ToF group required reconstruction surgery performed to the right branch pulmonary artery (PA) at the time of definitive repair. One patient required redo transannular patch surgery which was performed 2 years after the initial surgery. In follow-up, concomitant mild $(n=5)$ or moderate $(n=1)$ RVOT obstruction was noted in 6 patients.

\section{Cardiovascular Magnetic Resonance Imaging Protocol}

Magnetic resonance imaging was performed using a $1.5 \mathrm{~T}$ magnetic resonance imaging scanner (Avanto; Siemens Medical Systems, Erlangen, Germany).

Assessment of ventricular volumes and function using cine magnetic resonance imaging. Retrospective gated steady-state free precession cine magnetic resonance images of the heart were acquired in the vertical long-axis, 4-chamber view, and short-axis view covering the entirety of both ventricles (9-12 slices). Image parameters were as follows: $\mathrm{TR}=2.2 \mathrm{~ms} ; \mathrm{TE}=1.1 \mathrm{~ms}$; flip angle $=78$ degrees; slice thickness $=6-8 \mathrm{~mm}$; matrix $=192 \times 256$; field of view $=300-380 \mathrm{~mm}$; and temporal resolution $=40 \mathrm{~ms}$, acquired during a single breath-hold. Assessment of RV and left ventricular (LV) volumes was performed by manual segmentation of short-axis cine images with endocardial outline at end-diastole and end-systole (Argus, Siemens Medical Systems). End-diastolic and end-systolic volumes were calculated by use of Simpson's rule for each ventricle, and from these volumes the ejection fraction was calculated. RV mass was calculated via manual segmentation of the epicardial outline in end diastole. RVOT function was assessed from cine imaging (saggital views), and abnormal contraction was diagnosed if there was akinetic or aneurysmal formation where there was paradoxic contraction (defined as expansile anterior RVOT motion toward the sternum of $\geq 5 \mathrm{~mm}$ in ventricular systole). RVOT function was independently assessed by R.P. and P.L., who were each blinded to the diagnosis. Transannular plane systolic excursion (TAPSE) was calculated for the RV and measured in centimeters from the 4-chamber view in a fashion similar to that performed with 2-dimensional echocardiography. ${ }^{10}$

\section{Magnetic Resonance Flow Quantification}

PA flow data were acquired by use of a flow-sensitive gradient-echo sequence $(\mathrm{TR}=27 \mathrm{~ms} ; \mathrm{TE}=3.2 \mathrm{~ms}$; flip angle $=30$ degrees; slice thickness $=5 \mathrm{~mm}$; matrix $=256 \times 240$; temporal resolution $=30 \mathrm{~ms}$ ) during free breathing. Image planes were located at the midpoint of the main PA. ${ }^{11}$ Through-plane flow data were acquired by use of retrospective cardiac gating. Arterial blood flow was calculated from phase contrast images by use of a semiautomatic vessel edge-detection algorithm (Argus) with operator correction. Net forward flow within the main PA (milliliters) and PR fraction (\%) were calculated as total PA flow minus backward PA flow and percent backward PA flow over total PA flow, respectively.

\section{Three-Dimensional Magnetic Resonance Measurement of Pulmonary Artery Dimensions}

Measurements were acquired with an operator-independent isotropic 3dimensional balanced steady-state free precession whole heart imaging sequence. ${ }^{12}$ Imaging parameters were as follows: $\mathrm{TR}=1.6, \mathrm{TE}=3.2$, flip angle $=90$ degrees, and matrix $=152 \times 256$ to give an image resolution of $1.2 \times 1.2 \times 1.2 \mathrm{~mm}$. This is a navigator echo-gated sequence acquired during free respiration, with the navigator-echo window set around end expiration and a 6-mm acceptance window. ${ }^{13}$ Proximal, distal PA, and branch PA dimensions were measured.

The local research ethics committees approved the study, and all patients gave informed consent at the time of the clinically indicated cardiovascular magnetic resonance scan.

\section{Statistical Analysis}

All data are presented as mean \pm standard deviation or median and range. Statistical comparison of parametric data was performed with a 2-tailed unpaired Student $t$ test. The relationship between dichotomous variables was tested with the Fisher exact test. Statistical testing and data analysis were performed with GraphPad Prism Version 4.0 (GraphPad Software, San Diego, Calif).

\section{RESULTS}

Patient demographics are shown in Table 1. The ToF and PS groups were well matched for age at scan, age at repair surgery in ToF or initial valvotomy in PS, duration of exposure to PR (as estimated by the time from complete repair in ToF or initial valvotomy in PS), body surface area, heart rate, $\mathrm{PR}$ fraction, net forward PA flow, and main and branch PA dimensions. In the ToF group, 18 of 30 patients $(60 \%)$ 
TABLE 1. Patient demographics

\begin{tabular}{lccc}
\hline & ToF $(\mathbf{N}=\mathbf{3 0})$ & PS $(\mathbf{N}=\mathbf{3 0})$ & $\boldsymbol{P}$ value \\
\hline Age at scan (y) & $25.1 \pm 1.2$ & $26.6 \pm 1.8$ & NS \\
Gender & 18 male/ & 14 male/ & NS \\
& 12 female & 16 female & \\
Age at repair/valvotomy (y) & $2.8 \pm 0.4$ & $2.7 \pm 0.6$ & NS \\
Years exposed to PR & $22.0 \pm 0.9$ & $24.2 \pm 1.5$ & NS \\
Body surface area $\left(\mathrm{m}^{2}\right)$ & $1.79 \pm 0.03$ & $1.70 \pm 0.04$ & NS \\
Heart rate (beats/min) & $72.6 \pm 1.8$ & $74.1 \pm 3.3$ & NS \\
PR fraction $(\%)$ & $39.9 \pm 1.2$ & $36.6 \pm 1.2$ & NS \\
Net forward PA flow (ml) & $73.4 \pm 2.6$ & $74.6 \pm 2.8$ & NS \\
Main PA dimensions (mm) & $26.9 \pm 1.1$ & $26.7 \pm 0.8$ & NS \\
Right PA dimensions (mm) & $15.0 \pm 1.0$ & $17.4 \pm 1.1$ & NS \\
Left PA dimensions $(\mathrm{mm})$ & $17.5 \pm 1.1$ & $19.4 \pm 1.3$ & NS \\
\hline
\end{tabular}

$N S$, Not significant. Patients with repaired $\mathrm{ToF}(\mathrm{N}=30)$ are compared with patients with PS treated with valvotomy $(\mathrm{N}=30)$. Results are expressed as mean \pm standard deviation.

were male, whereas in the PS group 14 of 30 patients (47\%) were male.

The indexed ventricular volume data are shown in Figure 1. Indexed RV end-diastolic volumes were similar for both ToF and PS groups $\left(137 \pm 6 \mathrm{~mL} / \mathrm{m}^{2}\right.$ vs $128 \pm$ $\left.5 \mathrm{~mL} / \mathrm{m}^{2}, P=.25\right)$. However, indexed RV end-systolic volumes were higher in the ToF group when compared with the PS group $\left(70 \pm 5 \mathrm{~mL} / \mathrm{m}^{2}\right.$ vs $54 \pm 3 \mathrm{~mL} / \mathrm{m}^{2}$, $P=.008)$. Indexed LV volumes also demonstrated a similar pattern. LV end-diastolic volumes in patients with ToF were not significantly different from those in patients with PS $\left(72 \pm 2 \mathrm{~mL} / \mathrm{m}^{2}\right.$ vs $\left.67 \pm 2 \mathrm{~mL} / \mathrm{m}^{2}, P=.11\right)$, and indexed $\mathrm{LV}$ end-systolic volumes were higher in patients with ToF $\left(29 \pm 1 \mathrm{~mL} / \mathrm{m}^{2}\right.$ vs $\left.22 \pm 1 \mathrm{~mL} / \mathrm{m}^{2}, P<.001\right)$. Further subgroup analysis was performed among the percutaneously versus surgically treated patients in the PS group, with no statistically significant differences identified in LV or RV indexed volumes.

Changes in ventricular volumes were reflected in lower biventricular systolic function in the ToF group compared with the PS group. Specifically, the RV ejection fraction in the ToF group was $52 \% \pm 1.5 \%$ compared with $59 \% \pm 1 \%$ in the PS group $(P<.001)$. The LV ejection fraction in the ToF group was $61 \% \pm 1 \%$ compared with $67 \% \pm 1 \%$ in the PS group $(P<.001)$.

In regard to factors influencing function of the muscular component of the right ventricle, RV TAPSE was not significant between the ToF and PS groups $(2.05 \pm 0.08 \mathrm{~cm}$ vs $2.03 \pm 0.08 \mathrm{~cm}, P=.86)$. Further, RV mass was also similar between TOF and PS groups ( $95 \pm 5 \mathrm{~g}$ vs $81 \pm 6 \mathrm{~g}$, respectively, $P=.08$ ). RVOT function was significantly different between groups: The RVOT was considered contractile in 15 of 30 patients $(50 \%)$ with ToF and 28 of 30 of patients $(93 \%)$ with PS $(P=.0004)$ (Figure 2$)$. When patients with only contractile RVOTs were compared, there was no significant difference in indexed RV end-systolic
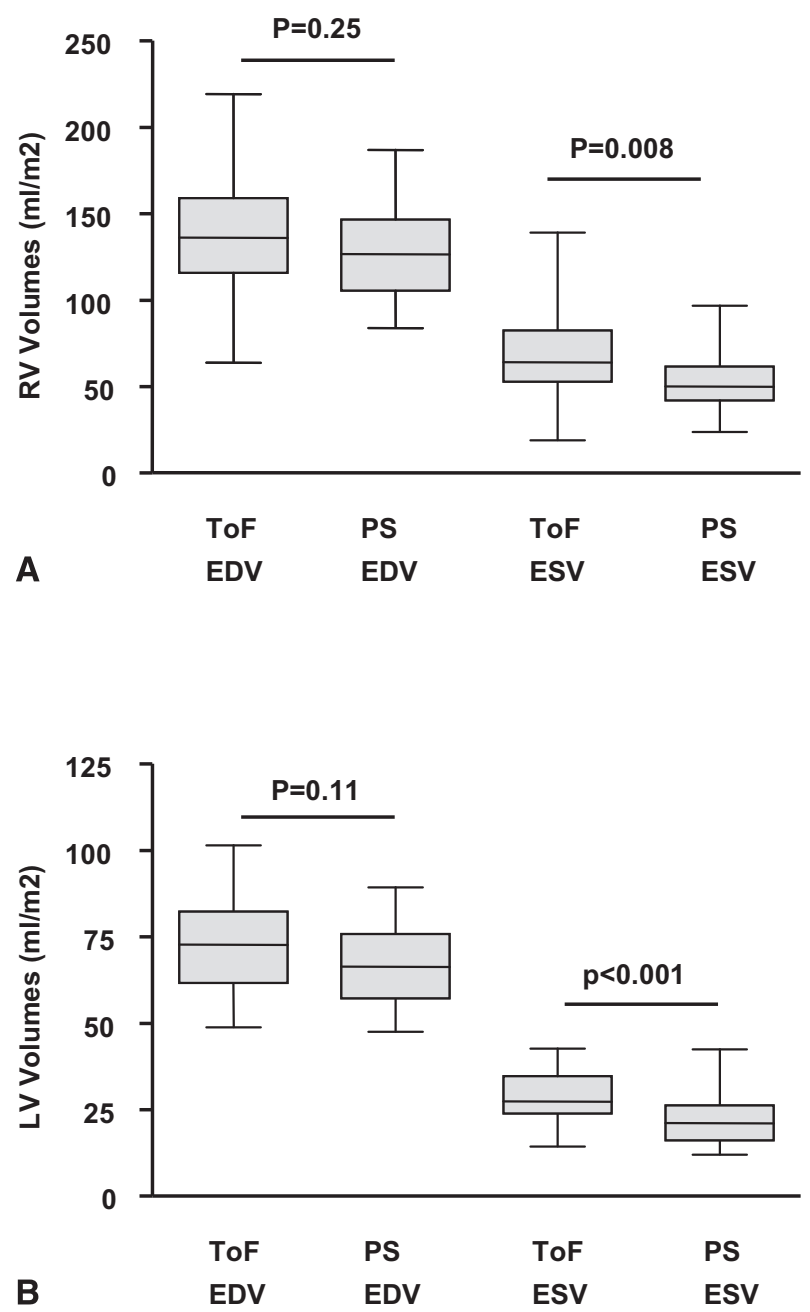

FIGURE 1. Indexed ventricular volumes. A, Indexed RV end-diastolic and end-systolic volumes for patients with repaired ToF and patients with PS treated with valvotomy. B, Indexed LV end-diastolic and endsystolic volumes for patients with ToF and PS. Results are expressed as mean \pm standard deviation. $R V$, Right ventricular; $T o F$, tetralogy of Fallot; $P S$, pulmonary stenosis; $E D V$, end-diastolic volume; $E S V$, end-systolic volume.

volumes $\left(63 \pm 6 \mathrm{~mL} / \mathrm{m}^{2}\right.$ vs $\left.54 \pm 3 \mathrm{~mL} / \mathrm{m}^{2}, P=.2\right)$ (Figure 3, $A)$ and RV ejection fraction $(55 \% \pm 2 \%$ vs $58 \% \pm 1 \%, P=.1$ ) (Figure 3, $B$ ) in patients with $\mathrm{ToF}$ or PS, respectively. Among patients with abnormal RVOT contractile function, all those with PS $(\mathrm{n}=2)$ were akinetic, whereas $60 \%$ were paradoxically contractile (aneurysmal) and $40 \%$ were akinetic in the ToF group $(\mathrm{n}=15)$. There was $100 \%$ agreement between blinded observers.

\section{DISCUSSION}

We have demonstrated that RVOT patch dysfunction in repaired ToF is responsible for higher end-systolic volumes and thus lower global measures of ventricular systolic function in both the RV and LV, which was observed in 


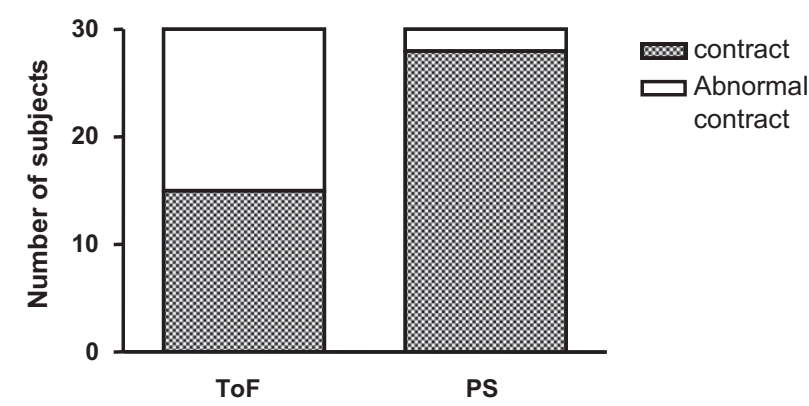

FIGURE 2. RVOT contractility was qualitatively compared between patients with repaired ToF and patients with PS treated with valvotomy. Patients in whom the RVOT was akinetic or contracted paradoxically (expansile in ventricular systole) were deemed abnormally contractile (clear panels). Patients with normally contractile RVOT are shown in the shaded panels. $R V$, Right ventricular; $L V$, left ventricular; $T o F$, tetralogy of Fallot; $P S$, pulmonary stenosis.

long-term follow-up. Thus, although there may be reasonable contractile function of the RV corpus as demonstrated by normal RV TAPSE, RV end-systolic volumes may remain elevated because of the akinetic or paradoxically contracting RVOT. This leads to a decrease in the effective forward flow and ejection fraction of the RV in these patients. Of note, this translates into decreased LV stroke volumes that results from higher end-systolic volumes of both ventricles, highlighting the complex interplay between the $\mathrm{RV}$ and LV that underpins long-term exercise capacity and prognosis. These findings were not evident in patients with PS treated with valvotomy, in whom there was a comparable amount of PR.

It is established that impaired LV systolic function combined with severe PR is known to influence exercise capacity and predict sudden cardiac death and atrial/ventricular arrhythmias in patients with $\mathrm{ToF}^{4-7}$ We identify RVOT patch dysfunction as an additional important factor in determining long-term LV volumes and function, and thus having implications for prognosis. In our study, although patients with PS had PR amounts that were comparable to those with ToF, biventricular volumes and function were more optimal in the PS group. The absence of RVOT patch dysfunction in PS may therefore lead to better functional outcomes despite similar loading conditions with severe PR. Further, those patients with ToF in whom the RVOT was contractile had similar favorable indices of ventricular function as observed in the patients with PS. Patients with ToF, and in particular those with RVOT patch dysfunction, were generally considered in the "lower normal" clinical range for RV and LV parameters. Serial data and correlation with cardiopulmonary exercise parameters are warranted to further understand the clinical implications of these findings.

Our data provide evidence that early repair strategies in ToF and PS have long-term hemodynamic sequelae. To

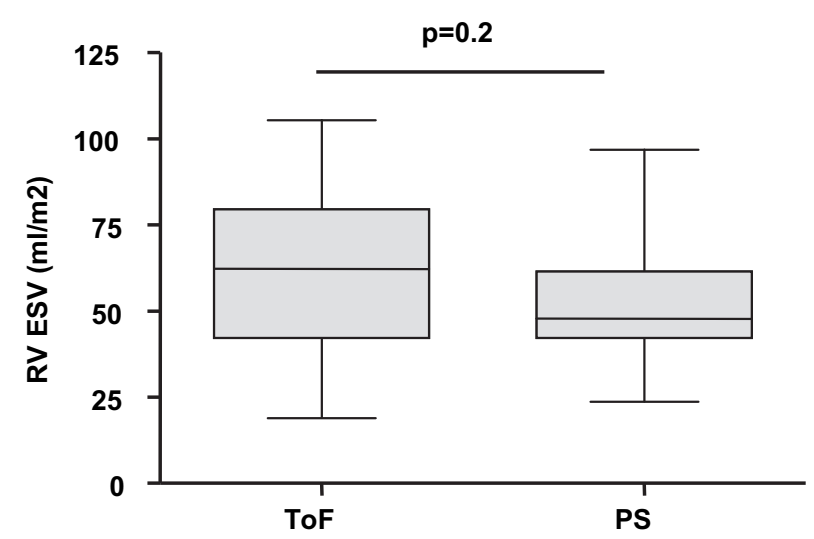

A

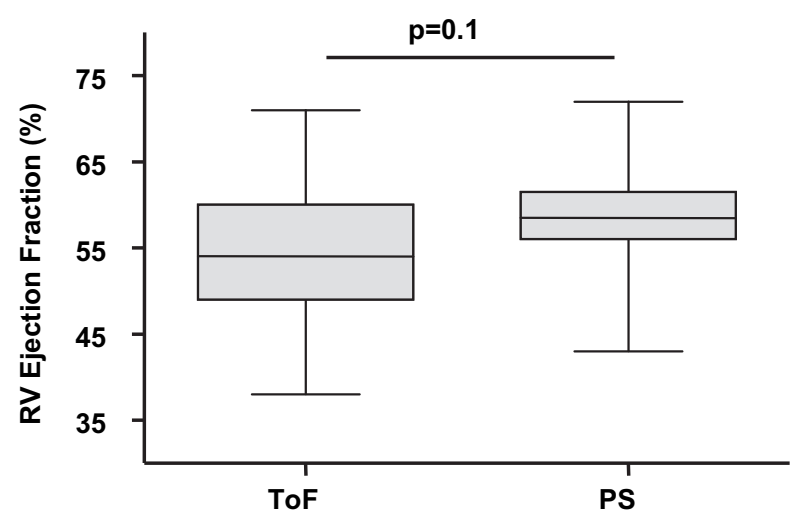

B

FIGURE 3. RV volumes and ejection fraction in patients with contractile RVOT. A, RV end-systolic volumes for patients with repaired ToF and patients with PS treated with valvotomy with contractile RVOT. B, RV ejection fraction for patients with ToF and PS with contractile RVOT. Results are expressed as mean \pm standard deviation. $R V$, Right ventricular; $E S V$, end-systolic volume; ToF, tetralogy of Fallot; $P S$, pulmonary stenosis.

improve hemodynamic outcomes when transannular patch surgery is deemed clinical necessary, we recommend the repair using a combined transatrial approach (to divide the septal and parietal trabeculations, and to patch the ventricular septal defect) and transpulmonary/infundibular approach (to complete division of RVOT muscular trabeculations if required), thereby preserving $\mathrm{RV}$ integrity by way of only limited infundibular incision $(1-2 \mathrm{~cm})$.

Optimal management strategies for chronic PR remain controversial. Recent reports highlight the importance of indexed ventricular volumes when setting the threshold to treat chronic PR. ${ }^{14-18}$ The particular emphasis of these guidelines is in the ability of volume-loaded, dilated right ventricles to "normalize" dimensions postsurgery. Our findings highlight the complexities involved in RV volume calculations. RVOT patch dysfunction significantly alters volumes despite good corpus function of the RV. Further, the heterogeneity between different congenital heart 
diseases that leads to similar amounts of PR in follow-up needs to be taken into consideration when devising treatment criteria and protocols.

\section{Study Limitations}

The limitations of this study include its retrospective nature and the relatively small numbers of patients within the groups. However, at present, the literature is lacking in studies examining the effects of chronic PR arising from different underlying pathologies apart from ToF. Also, we assume that the time of exposure to PR will approximate with the time from initial treatment (valvotomy or repair). The only way to confirm this would have been to perform prospective serial studies, and at the time many of our cohort were treated with current magnetic resonance imaging protocols that were not incorporated into clinical algorithms. Among our cohort, there were a small number of patients who were known to have mixed RVOT disease. Of note, in the PS surgically treated group, 4 patients had limited subannular RVOT patches, and contractile function was akinetic and not paradoxical in the 2 patients with reported dysfunction. Although these patients would have led to an underestimation of the volume/function differences between the groups, we think the inclusion of these patients more accurately reflects the cross-section of those encountered in clinical practice.

\section{CONCLUSIONS}

We have demonstrated that RVOT patch dysfunction in repaired ToF is responsible for higher end-systolic volumes and thus lower global measures of biventricular systolic function when compared with patients with PS (treated with valvotomy) and comparable amounts of residual PR. These observations highlight the importance of the initial repair surgery in ToF for late outcomes including alterations in LV volumes and function, which underpin long-term exercise capacity and prognosis.

\section{References}

1. Geva T, Sandweiss BM, Gauvreau K, Lock JE, Powell AJ. Factors associated with impaired clinical status in long-term survivors of tetralogy of Fallot repair evaluated by magnetic resonance imaging. J Am Coll Cardiol. 2004;43: 1068-74.
2. Niezen RA, Helbing WA, van der Wall EE, van der Geest RJ, Rebergen SA, de Roos A. Biventricular systolic function and mass studied with MR imaging in children with pulmonary regurgitation after repair for tetralogy of Fallot. Radiology. 1996;201:135-40.

3. Redington AN, Oldershaw PJ, Shinebourne EA, Rigby ML. A new technique for the assessment of pulmonary regurgitation and its application to the assessment of right ventricular function before and after repair of tetralogy of Fallot. $\mathrm{Br}$ Heart J. 1988;60:57-65.

4. Carvalho JS, Shinebourne EA, Busst C, Rigby ML, Redington AN. Exercise capacity after complete repair of tetralogy of Fallot: deleterious effects of residual pulmonary regurgitation. Br Heart J. 1992;67:470-3.

5. Gatzoulis MA, Balaji S, Webber SA, Siu SC, Hokanson JS, Poile C, et al. Risk factors for arrhythmia and sudden cardiac death late after repair of tetralogy of Fallot: a multicentre study. Lancet. 2000;356:975-81.

6. Marie PY, Marcon F, Brunotte F, Briancon S, Danchin N, Worms AM, et al. Right ventricular overload and induced sustained ventricular tachycardia in operatively "repaired" tetralogy of Fallot. Am J Cardiol. 1992;69:785-9.

7. Wessel HU, Bastanier CK, Paul MH, Berry TE, Cole RB, Muster AJ. Prognostic significance of arrhythmia in tetralogy of Fallot after intracardiac repair. Am J Cardiol. 1980;46:843-8.

8. Kopecky SL, Gersh BJ, McGoon MD, Mair DD, Porter CJ, Ilstrup DM, et al. Long-term outcome of patients undergoing surgical repair of isolated pulmonary valve stenosis. Follow-up at 20-30 years. Circulation. 1988;78:1150-6.

9. Roos-Hesselink JW, Meijboom FJ, Spitaels SE, vanDomburg RT, vanRijen EH, Utens EM, et al. Long-term outcome after surgery for pulmonary stenosis (a longitudinal study of 22-33 years). Eur Heart J. 2006;27:482-8.

10. Kaul S, Tei C, Hopkins JM, Shah PM. Assessment of right ventricular function using two-dimensional echocardiography. Am Heart J. 1984;107:526-31.

11. Rebergen SA, Niezen RA, Helbing WA, van der Wall EE, de Roos A. Cine gradient-echo MR imaging and MR velocity mapping in the evaluation of congenital heart disease. Radiographics. 1996;16:467-81.

12. Jhooti P, Gatehouse PD, Keegan J, Bunce NH, Taylor AM, Firmin DN. Phase ordering with automatic window selection (PAWS): a novel motion-resistant technique for 3D coronary imaging. Magn Reson Med. 2000;43:470-80.

13. Taylor AM, Jhooti P, Wiesmann F, Keegan J, Firmin DN, Pennell DJ. MR navigator-echo monitoring of temporal changes in diaphragm position: implications for MR coronary angiography. J Magn Reson Imaging. 1997;7: 629-36.

14. Buechel ER, Dave HH, Kellenberger CJ, Dodge-Khatami A, Pretre R, Berger F, et al. Remodelling of the right ventricle after early pulmonary valve replacement in children with repaired tetralogy of Fallot: assessment by cardiovascular magnetic resonance. Eur Heart J. 2005;26:2721-7.

15. Oosterhof $\mathrm{T}$, van Straten A, Vliegen HW, Meijboom FJ, van Dijk AP, Spijkerboer AM, et al. Preoperative thresholds for pulmonary valve replacement in patients with corrected tetralogy of Fallot using cardiovascular magnetic resonance. Circulation. 2007;116:545-51.

16. Therrien J, Provost Y, Merchant N, Williams W, Colman J, Webb G. Optimal timing for pulmonary valve replacement in adults after tetralogy of Fallot repair. $\mathrm{Am}$ J Cardiol. 2005;95:779-82.

17. Therrien J, Siu SC, McLaughlin PR, Liu PP, Williams WG, Webb GD. Pulmonary valve replacement in adults late after repair of tetralogy of Fallot: are we operating too late? J Am Coll Cardiol. 2000;36:1670-5.

18. Vliegen HW, van Straten A, de Roos A, Roest AA, Schoof PH, Zwinderman AH, et al. Magnetic resonance imaging to assess the hemodynamic effects of pulmonary valve replacement in adults late after repair of tetralogy of Fallot. Circulation. 2002;106:1703-7. 